\title{
ANALYSIS OF STABILITY DERIVATIVES IMPORTANT TO RECOVERY FROM SPIN
}

\author{
Marcin FIGAT $^{1}$, Zdobysław GORAJ ${ }^{2}$ \\ Aircraft Design Department, The Faculty of Power and Aeronautical Engineering, Warsaw University of \\ Technology, Nowowiejska 24, 00-665, Warsaw, POLAND \\ E-mails: ${ }^{1}$ mfigat@meil.pw.edu.pl (corresponding author); ${ }^{2}$ goraj@meil.pw.edu.pl
}

Received 25 September 2014; accepted 15 January 2016

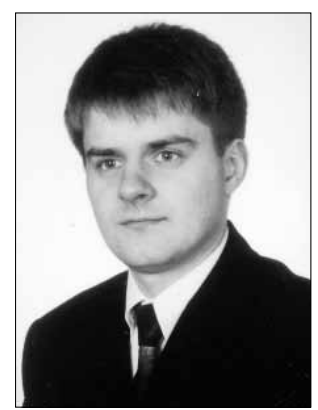

\section{Marcin FIGAT}

Marcin Figat is working as an assistant professor at Warsaw University of Technology, Department of Aircraft Design. He received the MSc degree in Aeronautical Engineering from the Faculty of Power and Aeronautical Engineering in 2002 and got PhD in 2009. He is interested in numerical aerodynamics, mechanics of flight and optimisation of aircraft design. He took part in a few projects of FP6/FP7 (NACRE, PPLANE) and many national research projects including SAMONIT (Mini UAV - a design and optimization for long endurance surveillance mission).

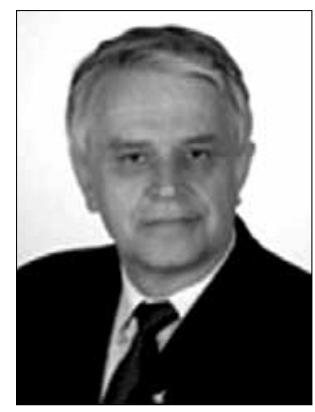

\section{Zdobysław GORAJ}

Aircraft designer and specialist in flight dynamics. His major topics of interest cover aircraft design and optimisation, stability and manoeuvrability of flying objects; aircraft performances and flight safety. Member of editorial boards of a few aeronautical journals including J. of Aerospace Engineering, J. of Aircraft Engineering and Aerospace Technology, Aviation and others. Initiator and main organiser of the International Seminar entitled: "Research and Education for Aircraft Design" (1994-2012). Doctor Honoris Causa of 2 universities: Moscow State Aviation Institute and Vilnius Gediminas Technical University, ICAS Programme Committee member, member of EASN Board of Directors, member of ACARE.

Abstract. This paper describes the numerical analysis of light aircraft stability derivatives in a wide range of angles of attack, important for recovery from spin. Stability derivatives versus angle of attack and sideslip were calculated using a CFD software, based on Euler equation combined with boundary layer equations. The analysis was performed up to the $40 \mathrm{deg}$ of angle of attack and up to $25 \mathrm{deg}$ of sideslip.

Keywords: aerodynamics, CFD, spin.

\section{Introduction}

Spin is known to be a very complex and still dangerous phenomenon in aviation. Certain types of new designed aircraft are incapable to recover from some types of spin, mainly due to the empennage design, moment of inertia and aerodynamic features. Such a situation is unacceptable, especially for light general-aviation airplanes which by nature are flown by not very experienced pilots. A huge number of papers and reports devoted to spin technology have been published (Goraj, Baron 2002; Baron,
Goraj 2001; Bowman 1971, Goraj et al. 2002; Goraj 2001), but there are still a number of challenges and questions which cannot be easily solved and answered for an individual design project and aircraft prototype. It is mainly because the spinning motion is very complicated and involves simultaneous rolling, yawing and pitching while the airplane is at high angles of attack and sideslip. Since it involves strongly separated flows in the region beyond the stall, the aerodynamic characteristics of the airplane are strongly nonlinear and time dependent. 
Numerical analysis of the spin should be made by a coupled CFD/Flight dynamics solver. Unfortunately, this method is very time-consuming. Therefore, we had to use one of the less-consuming methods of analysis for an aircraft's recovery from spin, which consists of an analysis of aircraft stability derivatives. Computations were made for a wide range of angles of attack and sideslip. This method allows making a preliminary assessment of the capabilities of aircraft recovery from spin.

\section{Basic Consideration}

The main goal of the study presented in the paper was to investigate the stability derivatives within a wide range of angles of attack and sideslip which are important for recovery from spin. A light aircraft in a classic configuration with a cruciform tailplane was selected as the object for this study. The layout of the aircraft considered is presented in Fig. 1.
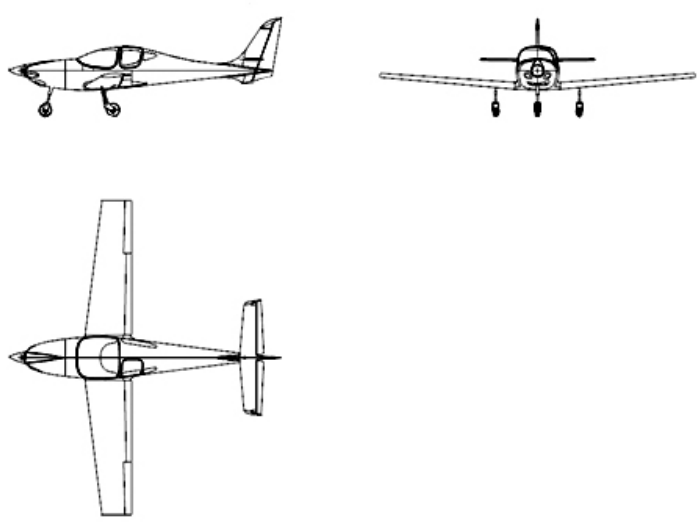

Fig. 1. Light aircraft

The whole work is focused mainly on the aerodynamic calculations of a light aircraft and its stability derivatives (Figat et al. 2013). The analysis starts from the creation of a CFD model. As a result of calculations, the aerodynamic characteristics versus the angle of attack and sideslip were obtained. Next, the change of the most important stability derivatives versus angle of attack and sideslip was investigated.

\section{Aerodynamic Analysis}

The preparation for aerodynamic calculations includes the creation of the CFD model, choice of the method of analysis and definition of a convention of the sing for the most important aerodynamic coefficients such as yaw moment coefficient $\mathrm{C}_{\mathrm{n}}$ and roll moment coefficient $\mathrm{C}_{\mathrm{l}}$. Moreover, conventions of sign connected with the rudder and elevator deflection were defined as well.

\subsection{The model}

Aerodynamic calculations were performed for the complete model of the aircraft which is presented in Fig. 2.
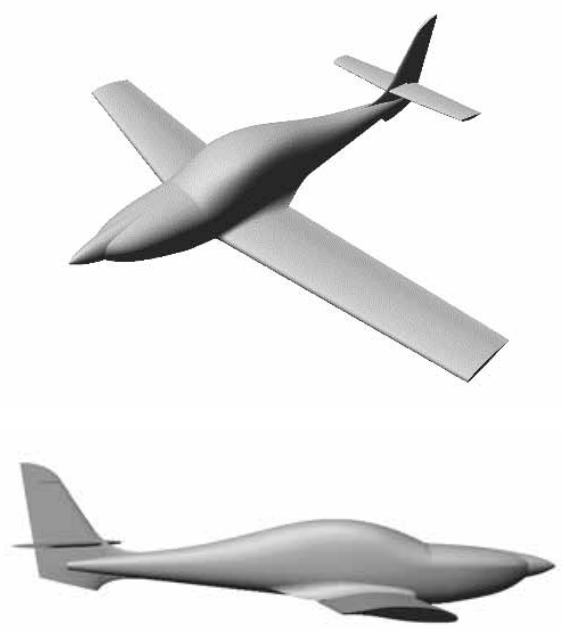

Fig. 2. The CFD model of a light aircraft

\subsection{Numerical method}

When selecting the software for numerical calculations one must remember that this software will be used at very high angles of attack, up to the $40 \mathrm{deg}$. It means we will enter overcritical angles of attack with complete flow separation on the vertical and horizontal stabilisers. Therefore, all computations were made by MGAERO (MGAERO user's manual) software. This software is based on the Euler equation (Anderson Jr. 1992) with a multi-grid acceleration scheme (Mavriplis 1992).The computational mesh is presented in Fig. 3.

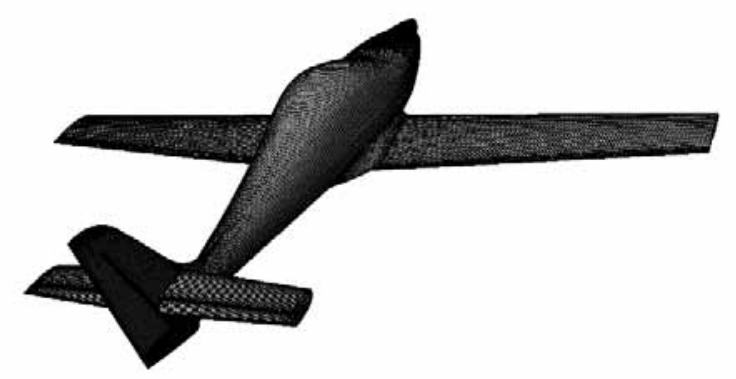

Fig. 3. Computational mesh

\subsection{Sign conventions}

Results of aerodynamic calculations were obtained by the aerodynamic solver presented in section 2.2. All the results were obtained in a specific coordinate system of the MGAERO software (MGAERO user's manual). According to it, the definition of sign convention is presented. Figs 4 and 5 present the positive value of aerodynamic coefficient $C_{n}$ for the yaw moment coefficient and $\mathrm{C}_{1}$ for the roll moment coefficient versus the sideslip angle. Both aerodynamic moment coefficients are referred to the semi-span of the wing. 


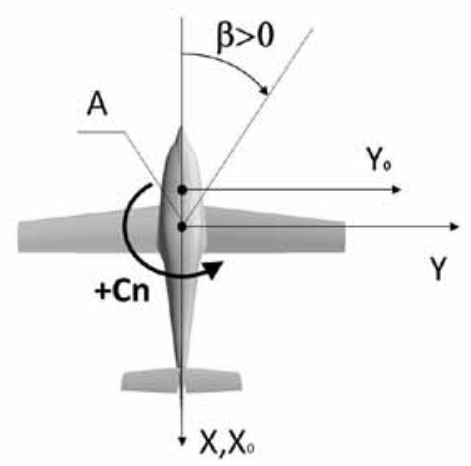

Fig. 4. Sign convention: yaw moment coefficient versus sideslip angle

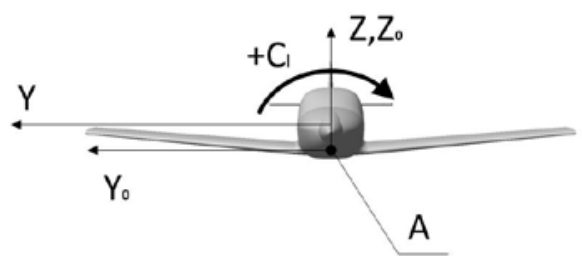

Fig. 5. Sign convention: rolling moment coefficient

According to the sign convention, the stability criterion for the spin recovery was established. The value (i.e. sign) of the stability derivatives which are the most important for the spin recovery should be satisfied by the following conditions:

$$
\frac{\partial C n}{\partial \beta}<0 ; \quad \frac{\partial C l}{\partial \beta}>0 ;
$$

It means that the derivative of the yaw moment with respect to the sideslip angle should be negative, but the derivative of the roll moment coefficient with respect to the sideslip angle should be positive. The fulfilment of both of these two conditions means that an aircraft has a satisfactory spin resistance.

\section{Results}

The computation schedule assumes that the basic aerodynamic characteristics for low angles of attack were obtained first. Next, the computation of stability derivatives was performed for the selected angles of attack and sideslip which correspond to the flight conditions in spin. The analysis was performed up to the $40 \mathrm{deg}$ of angle of attack and up to $25 \mathrm{deg}$ sideslip. Fig. 6. Cp distribution for the $\mathrm{a}=20 \operatorname{deg} \beta=0 \operatorname{degs} 6$ and Fig. 7. Cp distribution for $\mathrm{a}=40 \mathrm{deg}$ and $\beta=25 \mathrm{deg}$ present the $C_{p}$ distribution for the light aircraft considered.

\subsection{Results of computations}

The results of the computations of the aerodynamic coefficients: the side force, yaw moment and rolling moment, were obtained. The side force coefficient $\mathrm{C}_{\mathrm{s}}$ (Fig.

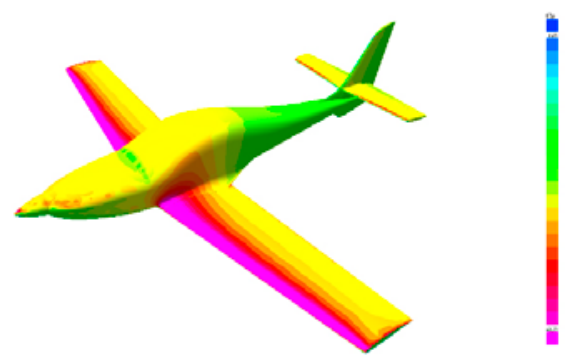

Fig. 6. Cp distribution for the $\alpha=20 \operatorname{deg} \beta=0 \operatorname{deg}$

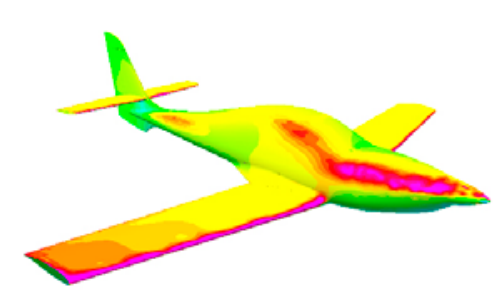

Fig. 7. Cp distribution for $\alpha=40 \mathrm{deg}$ and $\beta=25 \mathrm{deg}$

8 and Fig. 9) is presented first. The sign change is observed when the angle of attack is increasing. Next, the yaw moment coefficients $C_{n}$ versus the angle of attack and sideslip are presented in Figs 10 and 11. The roll moment coefficients $C_{1}$ courses are presented in Figs 12 and 13 .

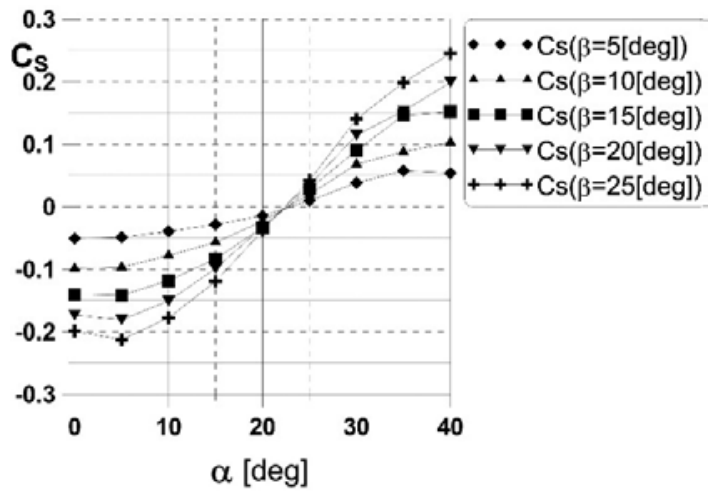

Fig. 8. Side force coefficient versus angle of attack

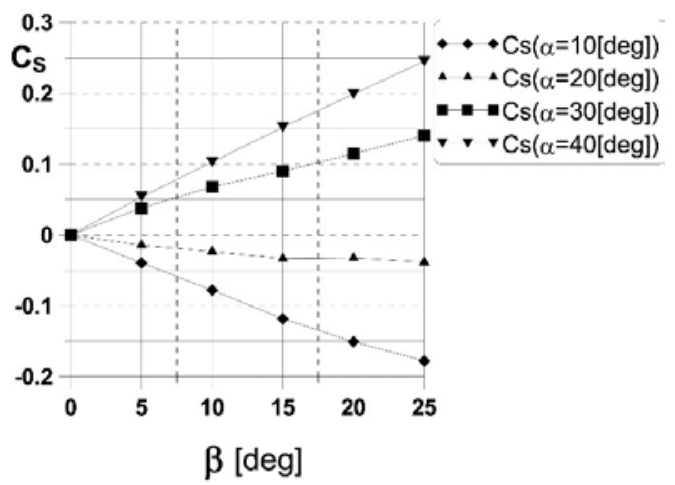

Fig. 9. Side force coefficient versus sideslip angle 


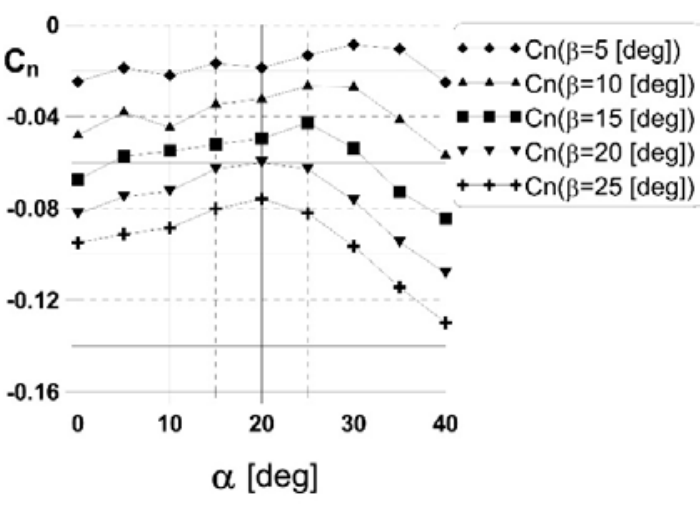

Fig. 10. Yaw moment coefficient $\mathrm{Cn}$ versus angle of attack

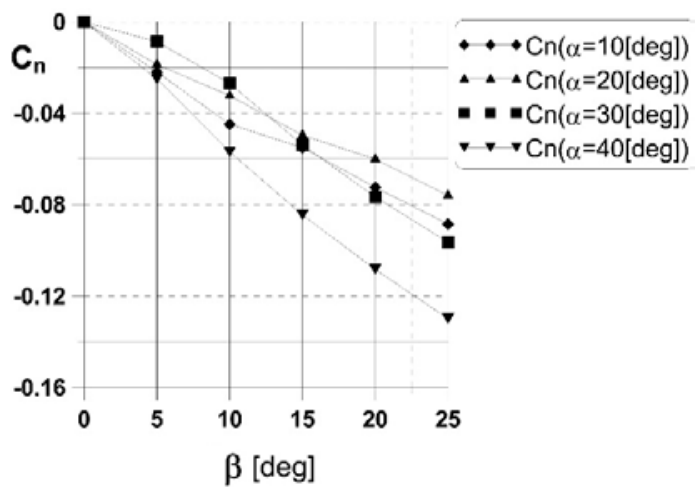

Fig. 11. Yaw moment coefficient $\mathrm{Cn}$ versus sideslip angle

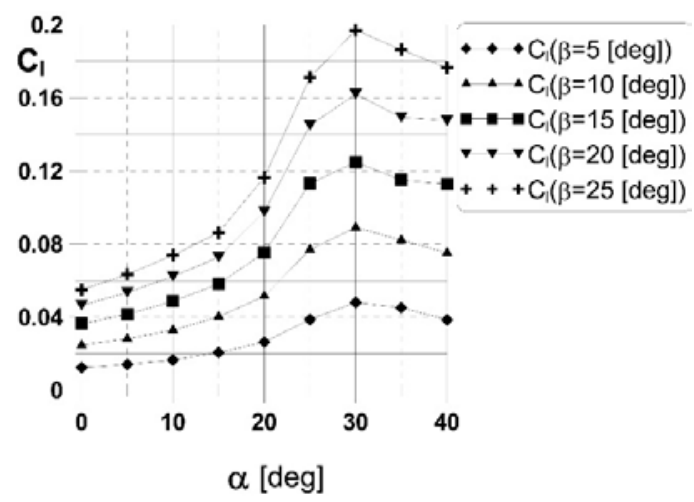

Fig. 12. Roll moment coefficient $\mathrm{Cl}$ versus angle of attack

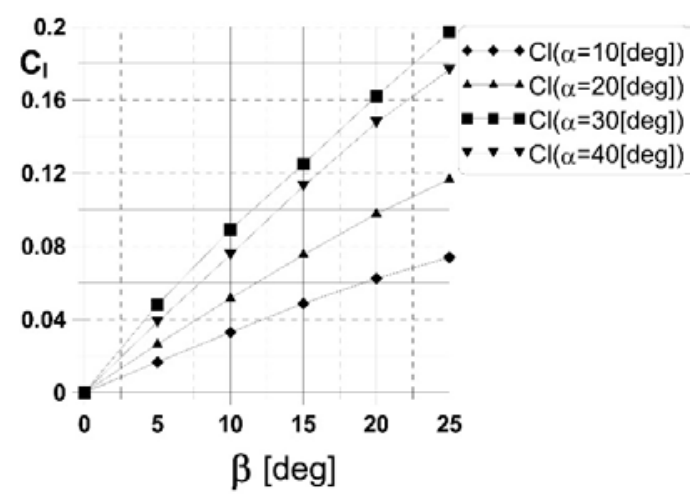

Fig. 13. Roll moment coefficient $\mathrm{Cl}$ versus sideslip angle
The results of the computations of aerodynamic coefficients reveal proper values and signs of force and moment coefficients (Figs 8-13). Only the side force coefficient changed the sign at higher angles of attack (about $22 \mathrm{deg}$ ).

\subsection{Stability derivatives}

The results of the aerodynamic calculations which were presented in section 3.1 allow to assess (calculate) the most important stability derivatives essential for an aircraft's behaviour and recovery from spin. Firstly, the efficiency of the vertical stabiliser versus the angle of attack was analysed (Fig. 14). This efficiency is represented by stability derivatives $\mathbf{a}_{\mathbf{v} 1}$. The side force coefficient generated at the vertical stabiliser with respect to the sideslip and angle of attack reveals that vertical stabiliser efficiency decreases in the considered range of angles of attack but its sign is still correct (i.e anti-spin properties are observed).

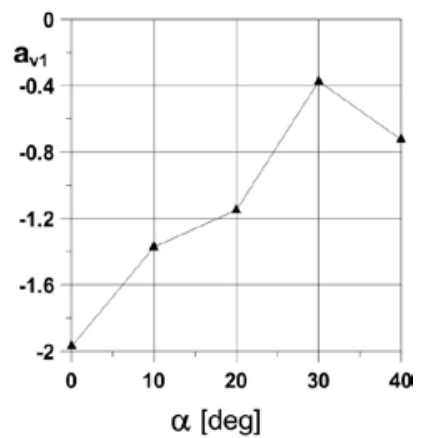

Fig. 14. Side force derivative of vertical stabiliser versus sideslip and angle of attack

Afterwards, the change in stability derivatives with respect to the sideslip was investigated (Figs 15-17). All the obtained results are acceptable (i.e. anti-spin properties are kept). The change in stability derivatives means that the aircraft's recovery from spin may be weak (poor), but the aircraft is, in theory, still stable. It means that its aerodynamic characteristics prevent the aircraft from entering into a spin. However, if the aircraft is spinning, these aerodynamic characteristics help to recover from the spin. Only the side force derivative changed the sign, but this stability derivative is less important for the dynamic behaviour of an aircraft in spin (i.e. spin is less sensitive to this derivative).

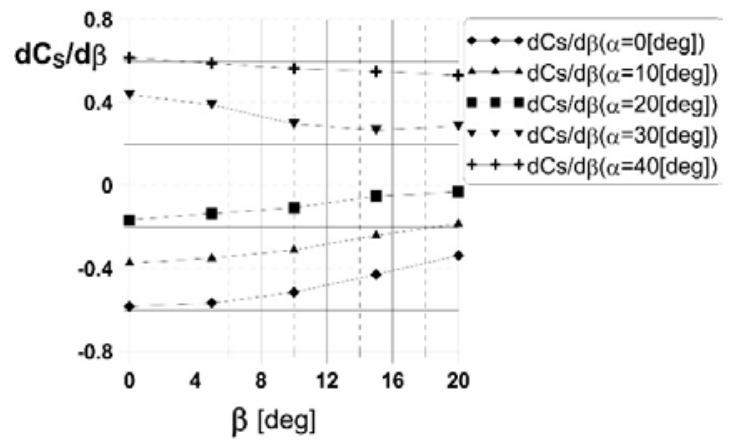

Fig. 15. Stability derivatives $\partial \mathrm{C}_{\mathrm{s}} / \partial \beta-$ side force coefficients versus sideslip angle 


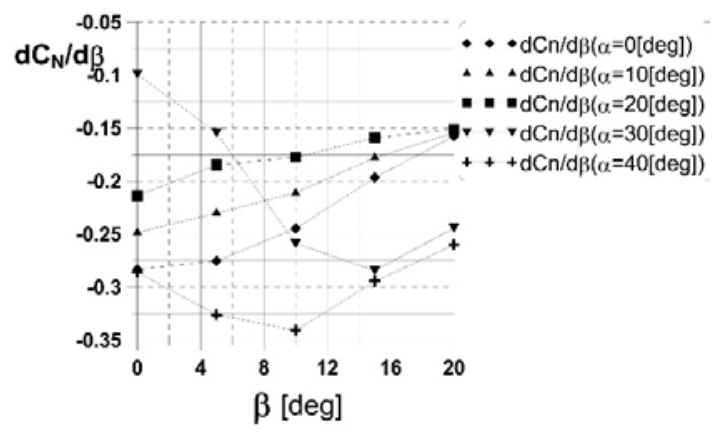

Fig. 16. Stability derivatives $\partial C_{n} / \partial \beta-$ yaw moment coefficients versus sideslip angle

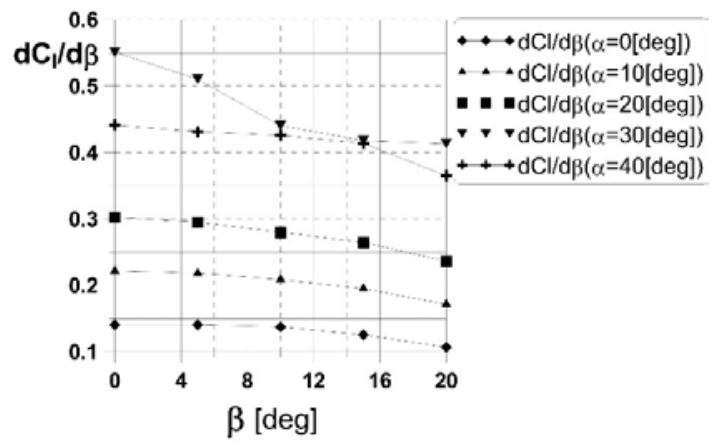

Fig. 17. Stability derivatives $\partial C_{1} / \partial \beta$ - roll moment coefficients versus sideslip angle

The results of computations of stability derivatives reveal that the aircraft is statically stable in the lateral direction. It means that the stability criterion is satisfied for the entire considered range of angles of attack and sideslips.

\section{Conclusions}

The paper presents the numerical analysis of light aircraft stability derivatives in a wide range of angles of attack important for recovery from spin. The analysis was performed up to $40 \mathrm{deg}$ of angles of attack and up to a 25 deg sideslip. The general conclusion that the aerodynamic analysis and stability derivative calculations revealed is that the aircraft under consideration is directionally stable in a wide range of angles of attack. This is caused by the fulfilment of the stability criterion, defined in section 2.3, in the entire range of angles of attack and sideslip. It means that its aerodynamic characteristics allow both protecting the aircraft against the casual entry into spin and enabling a relatively easy recovery if for any reason the aircraft has entered into a spin.

\section{References}

Anderson Jr., J. D. 1995. Computational fluid dynamics - the basis with applications. $1^{\text {st }}$ ed. McGraw-Hill.

Baron, A.; Goraj, Z. 2001. Analysis of the elevator and rudder efficiency in spin for an executive light aircraft, Transactions of the Institute of Aviation - Aeronautical Quarterly 167: 45-53 (in Polish).

Bowman, J. S. 1971. Summary of spin technology as related to light general aviation airplanes, NASA TN D-6575. Washington DC: NASA,.

Goraj, Z. 2001. Aerodynamics and flight dynamics of maneuverable airplanes with computational components, Scientific Library Series of the Institute of Aviation, vol. 13. Warsaw (in Polish).

Goraj, Z.; Baron, A. 2002. Theoretical, experimental and inflight spin investigations for an executive light airplane, in Proceedings of ICAS Congress, September 2002, Montreal. $523 \mathrm{p}$.

Goraj, Z.; Baron, A.; Kasprzyk, J. 2002. Dynamics of a light aircraft in spin, Aircraft Engineering and Aerospace Techno$\log y$ - An International Journal 2002 (in the press).

Figat, M.; Goraj, Z. 2013. Spin analysis of AT-5 aircraft with checking the requirements imposed by of CS-23, Report for AT Company (unpublished). Warsaw: Warsaw University of Technology.

MGAERO. A Cartesian multigrid Euler code for flow around arbitrary configurations, User's Manual Version 3.1.4.

Mavriplis, D. J. 1992. Three-dimensional unstructured multigrid for the Euler equations, Journal of Aircraft 30(7): 1753-1761. http://dx.doi.org/10.2514/3.11133 